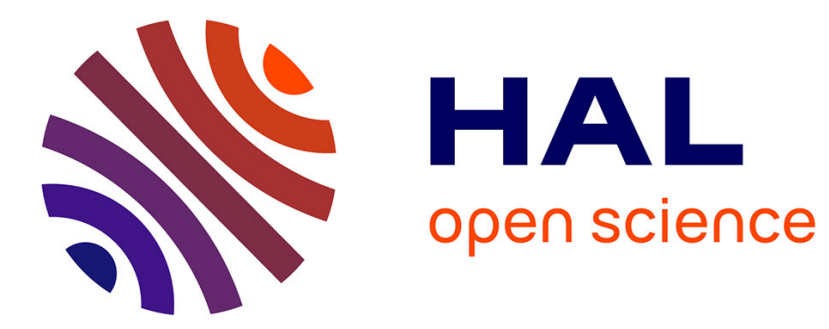

\title{
Image Retrieval using Fuzzy Representation of Colors
}

Amine Ait-Younes, Isis Truck, Herman Akdag

\section{To cite this version:}

Amine Aït-Younes, Isis Truck, Herman Akdag. Image Retrieval using Fuzzy Representation of Colors.

Soft Computing, 2007, 11 (3), pp.287-298. hal-00656854

\section{HAL Id: hal-00656854 \\ https://hal.science/hal-00656854}

Submitted on 5 Jan 2012

HAL is a multi-disciplinary open access archive for the deposit and dissemination of scientific research documents, whether they are published or not. The documents may come from teaching and research institutions in France or abroad, or from public or private research centers.
L'archive ouverte pluridisciplinaire HAL, est destinée au dépôt et à la diffusion de documents scientifiques de niveau recherche, publiés ou non, émanant des établissements d'enseignement et de recherche français ou étrangers, des laboratoires publics ou privés. 


\title{
Image retrieval using fuzzy representation of colors
}

\author{
Amine AÏT YOUNES ${ }^{1}$, Isis TRUCK ${ }^{2}$, Herman AKDAG ${ }^{1,3}$ \\ 1 CReSTIC-MODECO, Université de Reims Champagne Ardenne \\ 2 Labo IA, Université Paris 8 \\ 3 LIP6, Université Paris 6
}

Received: date / Revised version: date

\begin{abstract}
The problem addressed in this article is image indexing and retrieval according to the color. Indeed we propose a classification based on the dominant color(s) of the images. The process consists in two steps: first, assigning a colorimetric profile to the image in HLS space (Hue, Lightness, Saturation) and then, handling the query for the retrieval. To achieve the first step, the definition of hue is done using a fuzzy representation that takes into account the non-uniformity of color distribution. Then, lightness and saturation are represented through linguistic qualifiers also defined in a fuzzy way. Finally, the profile is built through fuzzy functions representing the membership degree of the image to different classes. Thus, the query for image retrieval is a pair (hue, qualifier). The second step looks for a match between the query and the profiles. In order to improve the software and to make it more flexible, the user can re-define the fuzzy representation of Hue, Lightness and Saturation, according to his own perception.
\end{abstract}

Keywords Image classification, dominant colors, $\mathrm{Hu}-$ man Computer Interaction, fuzzy membership functions, color spaces

\section{Introduction}

In this paper, we focus on the problem of color image retrieval and indexing.

Since the beginning of the digital era the image databases have grown and the need of retrieval has become more and more important. Thus, image retrieval problematic has been addressed thoroughly for several years [11], [18], [20] in many fields [13], [17], but many improvements are still needed.

\footnotetext{
* A. Aït Younes

rue des Crayères BP 1035, 51687 Reims cedex 2, France

e-mail: amine@leri.univ-reims.fr
}

There are multiple ways to retrieve images: one can look for a certain shape, texture, color, etc. As for us, we focus on the image retrieval according to the color. Indeed, in several medical applications such as tumor detection, the color is of great importance [8], [10]. For example, [8] addresses the problem of melanoma where the color of pigmented cells can determine whether the melanoma is malignant or not. Another field of application lies in web content detection: Hammami et al. classify images in determining whether they contain a lot of skin texture or not in order to detect adult and sexual contents [13].

There are many works that are based on the determination of a similarity degree between images. This kind of classification can be done through several techniques, for example: statistical approach like Support Vector Machines [1], [17], [29] and fuzzy logic [7], [19], [30], including fuzzy integrals [12], etc. Among these Image Retrieval Systems, we distinguish at least two kinds: those that consider the color histograms and those that don't. Barla et al. address the problem of classifying images by exploiting color and illumination features, using histogram intersections. The histogram intersection is used as a kernel function for SVMs and allows one to classify images by similarity of histograms [1]. Han \& Ma propose a fuzzy color histogram that permits to consider the color similarity across different bins and the color dissimilarity in the same bin [14]. Thus, as in [31], a pixel of a given color will contribute not only to its specific bin, but its membership value will be spread to other histogram bins.

Another kind of approach is presented by Wang \& Du: they propose an algorithm for indexing and retrieving images based on region segmentation, and they also compute similarities between images in order to classify them [32]. As for Frigui, he describes a system that offers the refinement of the user query [12]. The user's relevance feedbacks are modeled by fuzzy sets, i.e. the user expresses his satisfaction or discontentment by assigning a label to the retrieved images. A dissimilarity based on 
fuzzy integrals is then used. It is a kind of supervised learning for image retrieval systems.

All the aforementioned authors work with a query image. That is not the case of Binaghi et al. who use a user query expressed by crisp values of colors. More precisely, they provide methodological and technical solutions to compute similarities between the query and the image index. The user also has to choose the color dimension (hue, chroma or lightness), the image area covered by the referent color and the type of color distribution in the image. Thus, the user has to know exactly what he is looking for in terms of colors and color distribution in order to obtain satisfactory results. That is why we have focused on the problem of the query expression which is very simple in our case: the user can ask only for a certain tone if he wants.

The aim of our work is not to achieve a simple classification but to retrieve images according to their dominant color expressed through linguistic expressions and to adapt the classification to the user sensibility, since color perception is often very subjective. As Binaghi et al. did, we don't use a query image.

In the process we propose, choices for color spaces are important. Section 2 explains why a fuzzy representation of colors is needed while section 3 focuses on the determination for each new entry (image) in the database. Profiles are assigned to images and depend on the quantity of pixels or region of pixels that belong to color classes. The software we have developed and the statistical results are presented in section 4, then, some improvements to the processing are proposed. Especially, a system able to learn the user's color perception is introduced.

\section{How to represent color?}

\subsection{Color spaces}

As we know, one of the spaces usually used to represent the color on a screen is the RGB space (Red, Green, Blue). It is a three dimensional space representing the three primary colors that usually vary from 0 to 255 . The origin of this space $(0,0,0)$ corresponds to the lack of color which represents the "black" color. On the other hand the point $(255,255,255)$ corresponds to the maximum of color which represents the "white".

In classification methods, this space is often used in the calculation of similarity through color histogram [25] which represents the distribution of the pixels on the three axes (red, green, blue) (cf. figure 1). However with this kind of histogram it is difficult to define a fuzzy membership degree to a given color, for example how can one define in a fuzzy way a "pink" ?

Besides, the RGB space is not appropriate for our problem also because three dimensions (R, G and $B$ ) are necessary to identify a color. To facilitate the color

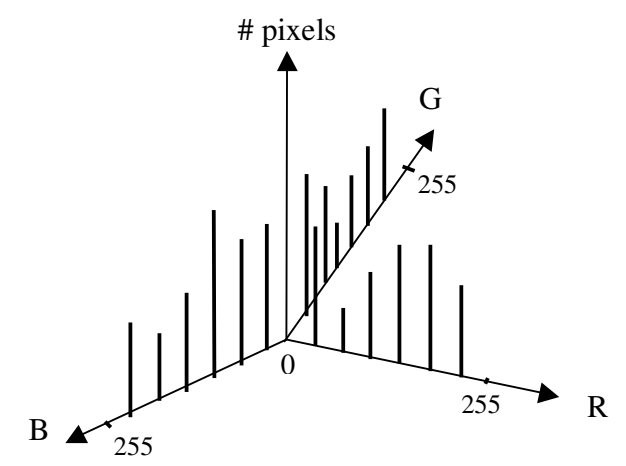

Fig. 1 An example of a color histogram.

identification we choose another space that allows us to characterize a color with only one dimension: its hue. Indeed hue is enough to recognize the color, except when the color is very pale or very somber. This other space is called HLS (Hue, Lightness, Saturation) where saturation corresponds to the quantity of "white" in the color and lightness corresponds to the light intensity of the color. The identification of color can be made in two steps : first H, then L, S. The HLS space can be represented through a cylinder or a bi-cone.

Moreover, in practice, various models of color representation also use a "two-step" identification of color. For example, Aron Sigfrid Forsius, Pantone Matching System, RAL (ReichsAusschuß für Lieferbedingungen und Gütesicherung), Munsell, ISCC-NBS (Inter-Society Color Council - National Bureau of Standards), etc. [9] use at first a color description by means of the hue then a refinement through the saturation and the lightness.

$\mathrm{H}$ is defined as an angle but we can also represent it in the interval $[0,255]$ as the other components L and S. The difference between $\mathrm{H}$ and the other components is that its definition interval loops which means that 0 and 256 are the same points. The "pure" red (represented in RGB space by the point $(255,0,0))$ corresponds to an angle equal to 0 for $h$, a saturation $s$ equal to 255 and a lightness $l$ equal to 128 .

Authors such as Hildebrand \& Reusch don't consider a "two-step" identification of the color. They define new color spaces, based on HSI color space (a variant of HLS space): HS (hue-saturation) color space and HI (hueintensity) color space [16]. In both spaces the third component (respectively $\mathrm{I}$ and $\mathrm{S}$ ) is set to the maximum value. Thus, a $3 \mathrm{D}$-membership function is assigned to a color (cf. figure 2).

Consequently, they are able to directly model colors such as dark blue or bright red. However, we have chosen to use the aforementioned "two-step" identification of the color with $\mathrm{H}$ first and then $\mathrm{L}$ and $\mathrm{S}$ because we think that, in our problem, the user may choose a global color such as blue, red, yellow... rather than a precise color such as dark blue, etc. We consider that the refinement of the query shall be performed in a second step. 


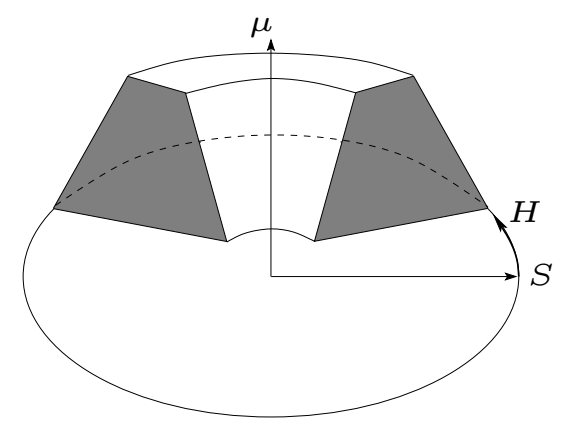

Fig. 2 3D-fuzzy set defined over the HS color space.

Our strategy is the following: we limit ourselves to the nine fundamental colors defined by the set $\mathcal{T}$ representing a good sample of colors (dimension $\mathrm{H}$ ) :

$\mathcal{T}=\{$ red, orange, yellow, green, cyan, blue, purple, magenta, pink\}

This set corresponds to the seven colors of Newton [21] to which we have added color pink and color cyan. This set is very intuitive since it approximately corresponds to the rainbow colors. Of course, this choice is not restrictive, we can modify the set of colors as desired.

\subsection{A fuzzy color representation}

Another interesting work is the Color Naming System proposed by Sugano [24]. The author describes a strategy to transform a crisp color (expressed with H, L and $\mathrm{S}$ ) into a set of words (hue name + tone modifier) expressing colors such as dark orange. He uses fuzzy membership functions to define saturation and lightness for a given hue. The hue space is composed of ten hues from the Japanese Industrial Standards. In contrast with this strategy, we start from linguistic expressions of colors (first, only hue, then hue + tone modifier) and we obtain a set of crisp values representing colors. However, like Sugano, we use fuzzy sets to define the $\mathrm{L}$ and $\mathrm{S}$ components.

As we have seen HLS space is convenient for our problem but it is a non UCS (uniform color scale) space [15], [27]. Indeed our eyes don't perceive small variations of hue when color is green $(h= \pm 85)$ or blue $(h= \pm 170)$ while they perceive them very well with orange $(h=21)$ for example. The easiest way would be to use UCS spaces such as CIELab, CIELuv, ... spaces. But they require more computational time to transform each pixel from RGB into these spaces, and mostly, they are unadapted to the user query because obtaining a tone modifier from these spaces is difficult [24].

To deal with non uniformly distributed scales, authors such as Herrera and Martínez propose to use fuzzy linguistic hierarchies with more or less labels, depending on the desired granularity [15]. Another approach from

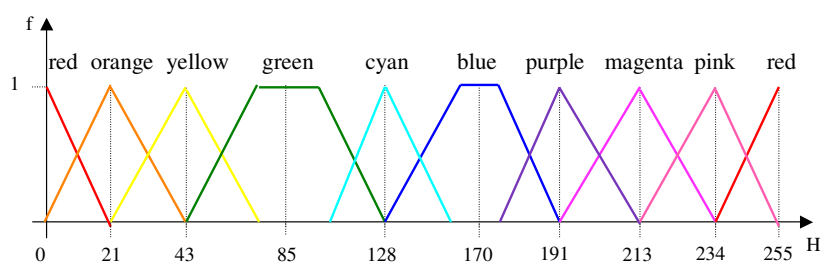

Fig. 3 The dimension $\mathrm{H}$.

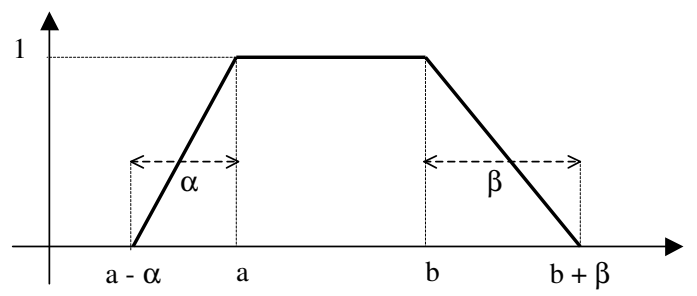

Fig. 4 Trapezoidal fuzzy subset.

Truck et al. is to represent the hues with trapezoidal or triangular fuzzy subsets thanks to color definitions from www.pourpre.com [26]. This technique is more empirical but fits the human perception better, that is why we also use this approach.

For each color of $\mathcal{T}$ they built a membership function varying from 0 to $1\left(f_{t}\right.$ with $\left.t \in \mathcal{T}\right)$. If this function is equal to 1 , the corresponding color is a "true color" (cf. figure 3$)$.

These functions were built using colors definition (www.pourpre.com). For each fundamental color, the associated interval is defined according to linguistic names of colors. For example to construct $f_{\text {yellow, we can use }}$ color "mustard" whose hue is equal to 55 and whose membership to $f_{\text {yellow }}$ is equal to \pm 0.5 .

For some colors, the result gives a wide interval. It is the case for the colors "green" and "blue" which are represented by trapezoidal fuzzy subsets.

For the construction of these functions, in this article we suppose that two functions representing two successive colors have their intersection point value equal to $1 / 2$. It means that when $h$ corresponds to an intersection point it can be assigned to both colors with the same weight.

As usual [3] we denote $(a, b, \alpha, \beta)$ a trapezoidal fuzzy subset (cf. figure 4). When the kernel is reduced to only one point, it is a triangular subset denoted by $(a, \alpha, \beta)$ since $a=b$.

Now we can define the membership function of any color $t$ : 


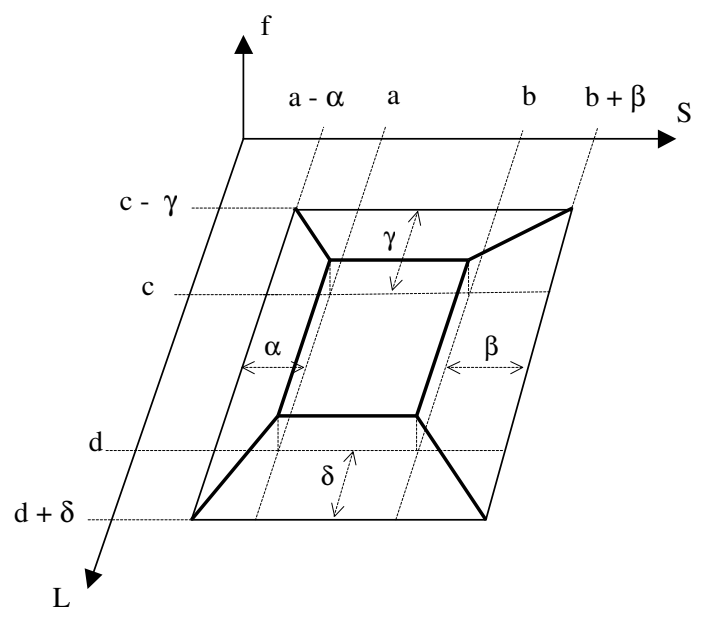

Fig. 6 Trapezoidal 3-D fuzzy subset.

$$
\forall t \in \mathcal{T}, f_{t}(h)= \begin{cases}1 & \text { if } h \geq a \\ & \wedge h \leq b \\ 0 & \text { if } h \leq a-\alpha \\ & \wedge h \geq b+\beta \\ \frac{h-(a-\alpha)}{\alpha} & \text { if } h>a-\alpha \\ & \wedge h<a \\ \frac{(b+\beta)-h}{\beta} & \text { if } h>b \\ & \wedge h<b+\beta\end{cases}
$$

Moreover if we want to complete the modelisation, it is necessary to take into account the other two dimensions $(\mathrm{L}, \mathrm{S})$. A scale representing the colorimetric qualifiers is associated to each dimension. These two intervals are divided into three: the first subinterval corresponds to a low value, the second to an average value and the last to a strong value. This division gives for saturation S: "dull", "moderately dull" and "saturated"; and for lightness L: "gloomy", "heavy" and "pallid".

These two scales are then aggregated to give nine qualifiers for colors defined by the following set (cf. figure 5) [26]:

$\mathcal{Q}=\{$ somber, dark, deep, gray, medium, bright, pale, light, luminous $\}$.

Each element of the set $\mathcal{Q}$ is associated to a membership function varying between 0 and $1\left(\tilde{f}_{q}\right.$ with $\left.q \in \mathcal{Q}\right)$. For these functions the intersection point value is also supposed equal to $1 / 2$ (cf. figure 7 ). Every function is represented through the set $(a, b, c, d, \alpha, \beta, \gamma, \delta)$ (cf. figure 6).

The membership function of any qualifier $q$ is defined below, i.e.: $\forall q \in \mathcal{Q}$,

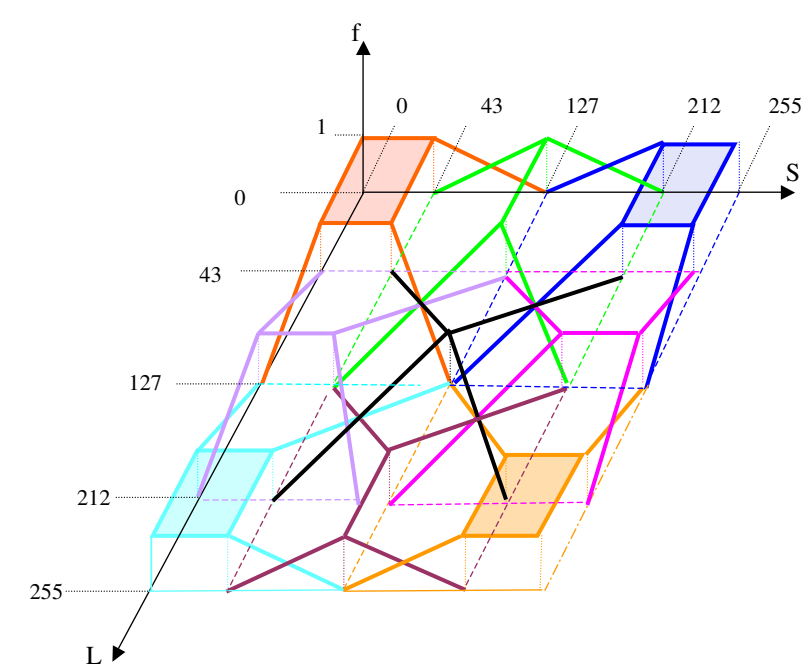

Fig. 7 Dimensions L and S.

$$
\tilde{f}_{q}(l, s)= \begin{cases}1 & \text { if } a \leq s \leq b \\ & \wedge c \leq l \leq d \\ & \text { if } s \leq a-\alpha \vee s \geq b+\beta \\ & \vee l \leq c-\gamma \vee l \geq d+\delta \\ \frac{l-(c-\gamma)}{\gamma} \quad & \text { if } c-\gamma<l<c \\ & \wedge \alpha l-\gamma s \leq \alpha c-\gamma a \\ & \wedge \beta l+\gamma s \leq \beta c+\gamma b \\ \frac{(d+\delta)-l}{\delta} & \text { if } d<l<d+\delta \\ & \wedge \beta l-\delta s>\beta d-\delta b \\ & \wedge \alpha l+\delta s>\alpha d+\delta a \\ \frac{s-(a-\alpha)}{\alpha} & \text { if } a-\alpha<s<a \\ & \wedge \alpha l-\gamma s>\alpha c-\gamma a \\ & \wedge \alpha l+\delta s \leq \alpha d+\delta a \\ \frac{(b+\beta)-s}{\beta} & \text { if } b<s<b+\beta \\ & \wedge \beta l+\gamma s>\beta c+\gamma b \\ & \wedge \beta l-\delta s \leq \beta d-\delta b\end{cases}
$$

We also took into account the colors black, gray and white. Fuzzy membership functions are associated to these colors $\left(f_{\text {black }}, f_{\text {white }}\right.$ and $\left.f_{\text {gray }}\right)$. These colors are completely defined through the spaces L and S. If the lightness is very low then the color is white, and a high level of the lightness means the color is black. If the saturation is very low then the color is gray. For this last color we define three qualifiers : dark, medium and light which are associated to fuzzy membership functions: $\tilde{f}_{\text {dark }}, \tilde{f}_{\text {medium }}$ and $\tilde{f}_{\text {light }}$ (cf. figure 8).

\section{Image processing}

The image processing aims at determining a profile corresponding to the various categories: the nine fundamental colors and the nine color qualifiers. For each pixel of 


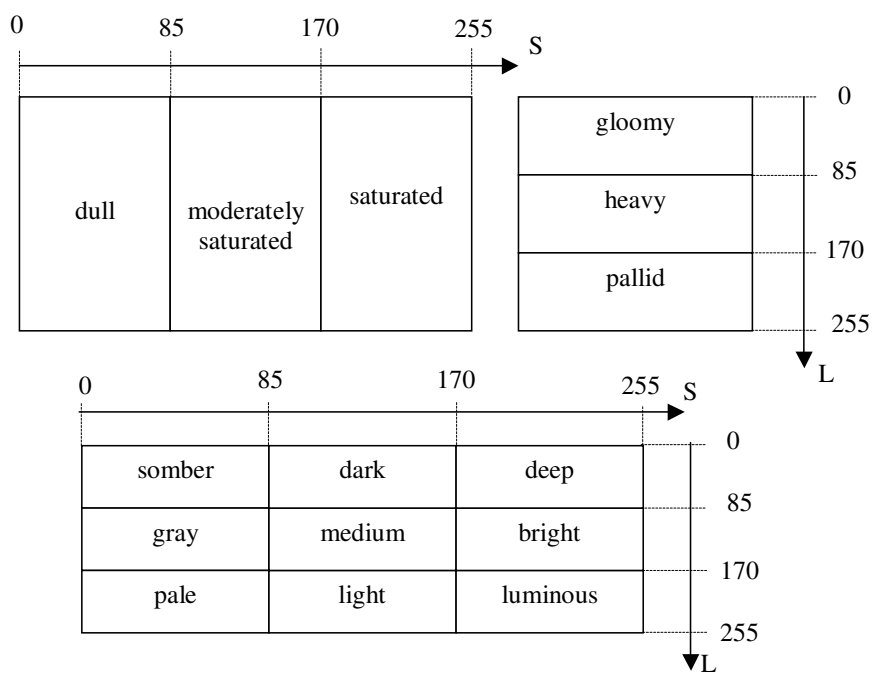

Fig. 5 Fundamental color qualifiers.

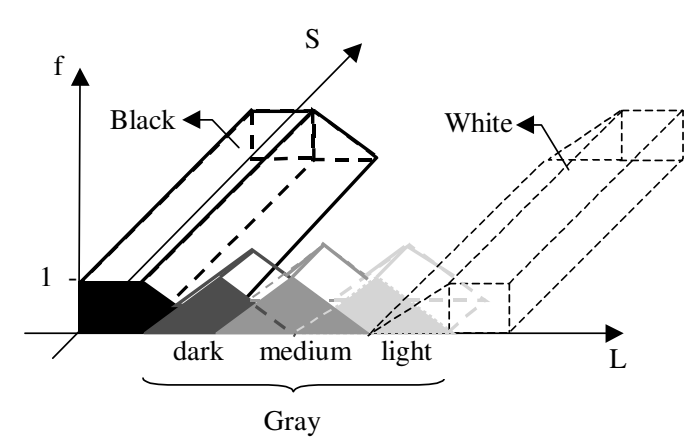

Fig. 8 Black, gray and white

the image we can determine the values taken by the various membership functions of the categories. For each category the value obtained corresponds to the ratio between the sum, on all the pixels of the image, of the membership functions values and the number of pixels, which gives a quantity between 0 and 1 . This quantity is the membership degree of an image to the given class.

The membership degree of an image to a certain class is defined as follow:

Let $I$ be an image.

Let $\mathcal{P}$ be the set representing the pixels of $I$

Each element $p$ of the set $\mathcal{P}$ is defined by its color coordinates $\left(h_{p}, l_{p}, s_{p}\right) . p$ can be one pixel or a set of pixels. We can calculate the functions $f_{t}\left(h_{p}\right), \tilde{f}_{q}\left(l_{p}, s_{p}\right)$ for $t \in \mathcal{T}$ and $q \in \mathcal{Q}$.

Let $F_{t}$ and $\widetilde{F}_{t, q}$ be the following functions, representing the membership degree of $I$ to the classes $t$ and $(t, q)$ :

$$
\begin{aligned}
-F_{t}(I) & =\frac{\sum_{p \in \mathcal{P}} f_{t}\left(h_{p}\right)}{|\mathcal{P}|} \forall t \in \mathcal{T} \\
-\widetilde{F}_{t, q}(I) & =\frac{\sum_{p \in \mathcal{P}} \tilde{f}_{q}\left(l_{p}, s_{p}\right) \times g_{t}\left(h_{p}\right)}{|\mathcal{P}|} \\
\forall(t, q) & \in \mathcal{T} \times \mathcal{Q}
\end{aligned}
$$

with $g_{t}\left(h_{p}\right)=\left\{\begin{array}{l}1 \text { if } f_{t}\left(h_{p}\right) \neq 0 \\ 0 \text { else }\end{array}\right.$

Example 1 Let us consider only 2 pixels $p_{0}$ and $p_{1}$ to simplify.

$$
\begin{gathered}
p_{0}:\left\{\begin{array}{l}
h_{p_{0}}=178 \\
l_{p_{0}}=50 \\
s_{p_{0}}=100
\end{array}, p_{1}:\left\{\begin{array}{l}
h_{p_{1}}=173 \\
l_{p_{1}}=255 \\
s_{p_{1}}=128
\end{array}\right.\right. \\
-f_{\text {blue }}\left(h_{p_{0}}\right)=f_{\text {blue }}(178)=0.9 \\
\tilde{f}_{\text {somber }}\left(l_{p_{0}}, s_{p_{0}}\right)=\tilde{f}_{\text {somber }}(50,100)=0.34 \\
-f_{\text {blue }}\left(h_{p_{1}}\right)=1, \tilde{f}_{\text {somber }}\left(l_{p_{1}}, s_{p_{1}}\right)=0
\end{gathered}
$$

So, for class "blue" the value shall be:

$F_{\text {blue }}(I)=0.95$

And for class "somber" from "blue", the value shall be: $\widetilde{F}_{\text {blue,somber }}(I)=0.17$

Every image is defined by a profile of 96 elements $(|T|+|T \times Q|+\mid\{$ black, white, gray $\}|+|\{$ gray $\} \times$ $\{$ dark, medium, light $\} \mid=9+81+3+3)$. A profile can be presented as follows :

$$
\left[F_{t}(I), \widetilde{F}_{t, q}(I)\right]
$$

An image can be assigned to several classes, there are 96 classes, 12 principal : $C_{t}$ with $t \in \mathcal{T} \cup\{$ black, white, gray $\}$, and 84 subclasses which correspond to a refinement of the research: $\widetilde{C}_{t, q}$ with $(t, q) \in \mathcal{T} \times \mathcal{Q} \cup\{$ gray $\} \times$ $\{$ dark, medium, light $\}$.

As shown in figure 9 the classes can be represented through a tree with father-son relationship, the classes $C_{t}$ with $t \in \mathcal{T}$ can be considered as fathers and the classes $\widetilde{C}_{t, q}$ with $(t, q) \in \mathcal{T} \times \mathcal{Q}$ as their sons. For example the father class of class $\widetilde{C}_{\text {red,somber }}$ is $C_{r e d}$.

Let us denote:

- $F^{*}(I)=\max _{t \in T}\left(F_{t}(I)\right)$

- $\widetilde{F}_{t}^{*}(I)=\max _{q \in Q}\left(\widetilde{F}_{t, q}(I)\right) \forall t \in T$, and for $t=$ gray, $q \in\{$ dark, medium, light $\}$ 


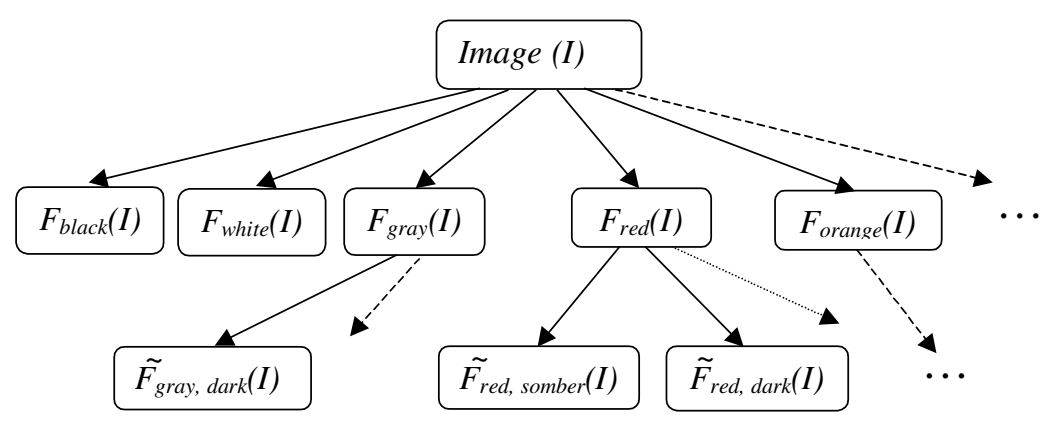

Fig. 9 Profile representing an image.

An image $I$ will be assigned to:

- the classes $C_{t}$ if $F_{t}(I) \geq F^{*}(I)-\lambda, \forall t \in \mathcal{T} \cup\{$ black, white, gray\},

with $\lambda$ a tolerance threshold.

- the classes $\widetilde{C}_{t, q}$ if $F_{t}(I) \geq F^{*}(I)-\lambda$ and $\widetilde{F}_{t, q}(I) \geq$ $\widetilde{F}_{t}^{*}(I)-\lambda, \forall(t, q) \in \mathcal{T} \times \mathcal{Q} \cup\{$ gray $\} \times\{$ dark, medium, light\}.

Thus, an image can be assigned to several classes, and it can be assigned to a subclass only if it is also assigned to its father class. For example, an image cannot be assigned to "red, bright" class $\left(\widetilde{C}_{\text {red,bright }}\right)$ if it is not assigned to the "red" one $\left(C_{r e d}\right)$.

Now we can present the software itself developed in $\mathrm{C}++$.

\section{Our software}

\subsection{Presentation}

Information concerning the images are stored in a database (cf. figure 10). That helps us to optimize the exploitation of these information. Each image will be represented through its previously defined profile.

We associate a table to each kind of classes, one representing the 9 principal classes (table "Image-hue") and another for the subclasses (table "Image-qualifier"). Two other tables ("Hue", "Qualifier") will facilitate any modification of the sets $\mathcal{T}$ and $\mathcal{Q}$ (cf. figure 10).

The software is divided into two sections, the first one corresponds to the treatment and the insertion of the images in the database, the second one to the exploitation of this database through requests with linguistic terms (cf. figure 11).

The image processing aims at building its profile. In the first section, a window allows us to select and display the image to be inserted in the database. Once the image is inserted the software displays all the stored images.

In the second section, the user of the software has the possibility of carrying out research on two levels. The first one corresponds to the nine fundamental colors (dimension $\mathrm{H}$ ), the second one to the nine color qualifiers.
Indeed, once the Hue is selected, the user has the possibility to refine his request by specifying a color qualifier. For that, it is enough for him to choose one proposed in the list, or to click on the corresponding zone in the image. For example, figure 12 shows the images whose dominant color is "luminous blue".

When a pair (color, qualifier) is chosen, the software proposes linguistic names of colors (expressed in natural language) that correspond to the choice: e.g. "cornflowerblue", "aliceblue", "lightskyblue" for a "luminous blue". Actually, it can be easier for some users to work with linguistic names of colors than with a pair (color, qualifier). In table 1, we present several linguistic names of blue classified according to the nine qualifiers. The chosen dictionary is HtmlColors, but any other dictionary can be used (e.g. Pantone, Munsell, etc.)

\begin{tabular}{|l|l|l|}
\hline $\begin{array}{l}\text { somber } \\
\text { darkslategray }\end{array}$ & $\begin{array}{l}\text { dark } \\
\text { midnightblue }\end{array}$ & $\begin{array}{l}\text { deep } \\
\text { darkblue }\end{array}$ \\
\hline $\begin{array}{l}\text { gray } \\
\text { slategray } \\
\text { cadetblue }\end{array}$ & $\begin{array}{l}\text { medium } \\
\text { darkslateblue } \\
\text { slateblue } \\
\text { steelblue }\end{array}$ & $\begin{array}{l}\text { bright } \\
\text { mediumblue } \\
\text { royalblue } \\
\text { dodgerblue }\end{array}$ \\
\hline $\begin{array}{l}\text { pale } \\
\text { silver } \\
\text { lightsteelblue }\end{array}$ & $\begin{array}{l}\text { light } \\
\text { lavander } \\
\text { lightblue }\end{array}$ & $\begin{array}{l}\text { luminous } \\
\text { cornflowerblue } \\
\text { aliceblue } \\
\text { lightskyblue }\end{array}$ \\
\hline
\end{tabular}

Table 1 Linguistic names for color blue.

Two other kinds of requests are handled: the first one allows us to retrieve $\mathrm{B} \& \mathrm{~W}$ and gray-level images (in this case, $H$ is not considered), and the second one allows us to retrieve images with more than one dominant color. One-color requests can be successively added (composed) to obtain a multi-color request (cf. figure 13).

\subsection{Statistical results}

For this kind of research, a comparison with the other similar works is usually welcome. But this is a hard 


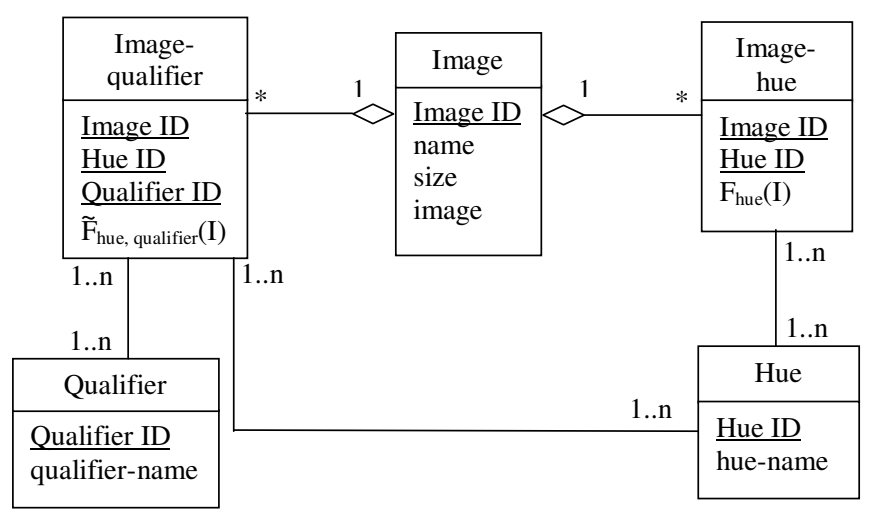

Fig. 10 Class diagram of the database.

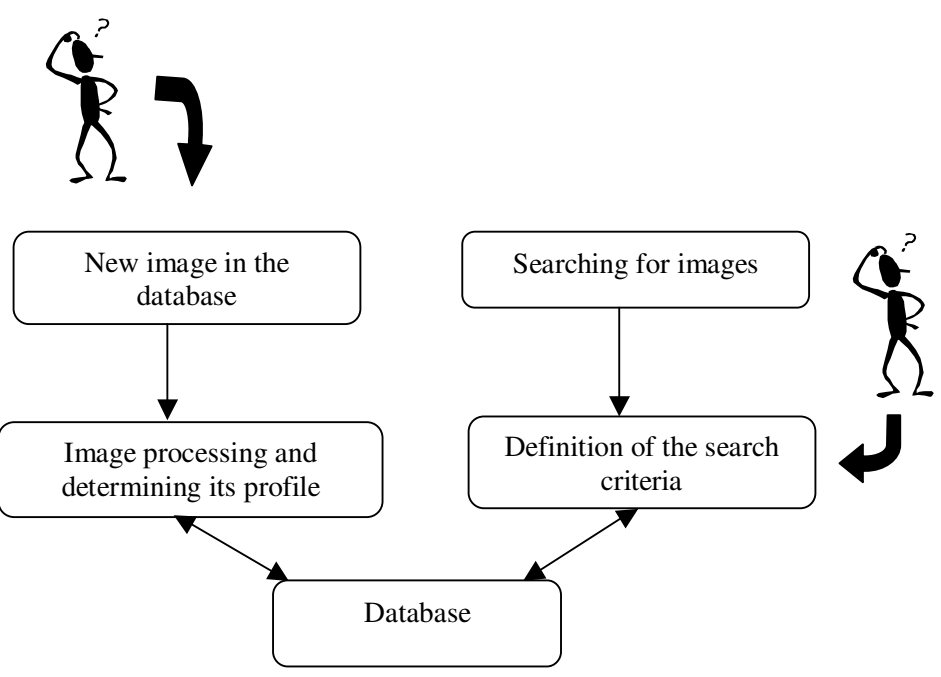

Fig. 11 Human-computer interaction.

task for several reasons. First, most of the research includes an image query which we don't, as we focus on a simple color query. Secondly, the image databases are usually not the same. Indeed, some authors use texture databases [2], [12] while some others use Comstock database [31], or Common Color Dataset [14], etc. However, authors like Omhover et al. propose their software online (http://strict.lip6.fr) which is very convenient for a comparison. But they use an image query and they compare not only colors but also shapes in the image, which can't be compared to our method. That is why we only propose a performance study of our software.

As Salton and McGill proposed in [22] the performance of a software can be evaluated in two steps:

1. Response time: it represents the time elapsed between the user query and the software response. For all the works using an image query, this information is important. However in our work the image processing is done before the image retrieval (cf. figure 11).
2. Recall and precision: images are considered either relevant or not relevant, and retrieved or not retrieved according to the request (cf. table 2).

\begin{tabular}{c|c|c} 
& Relevant & Not Relevant \\
\hline Retrieved & $\begin{array}{c}\text { RR } \\
\text { (correctly retrieved) }\end{array}$ & $\begin{array}{c}\text { RNR } \\
\text { (falsely retrieved) }\end{array}$ \\
\hline Not Retrieved & $\begin{array}{c}\text { NRR } \\
\text { (missed) }\end{array}$ & $\begin{array}{c}\text { NRNR } \\
\text { (correctly rejected) }\end{array}$ \\
\hline
\end{tabular}

Table 2 Performance of a software.

Recall measures the ability of the software to retrieve all relevant images:

$$
\text { Recall }=\frac{\text { relevant retrieved }}{\text { all relevant }}=\frac{\mathrm{RR}}{\mathrm{RR}+\mathrm{NRR}}
$$

and precision measures the ability to retrieve only relevant images: 


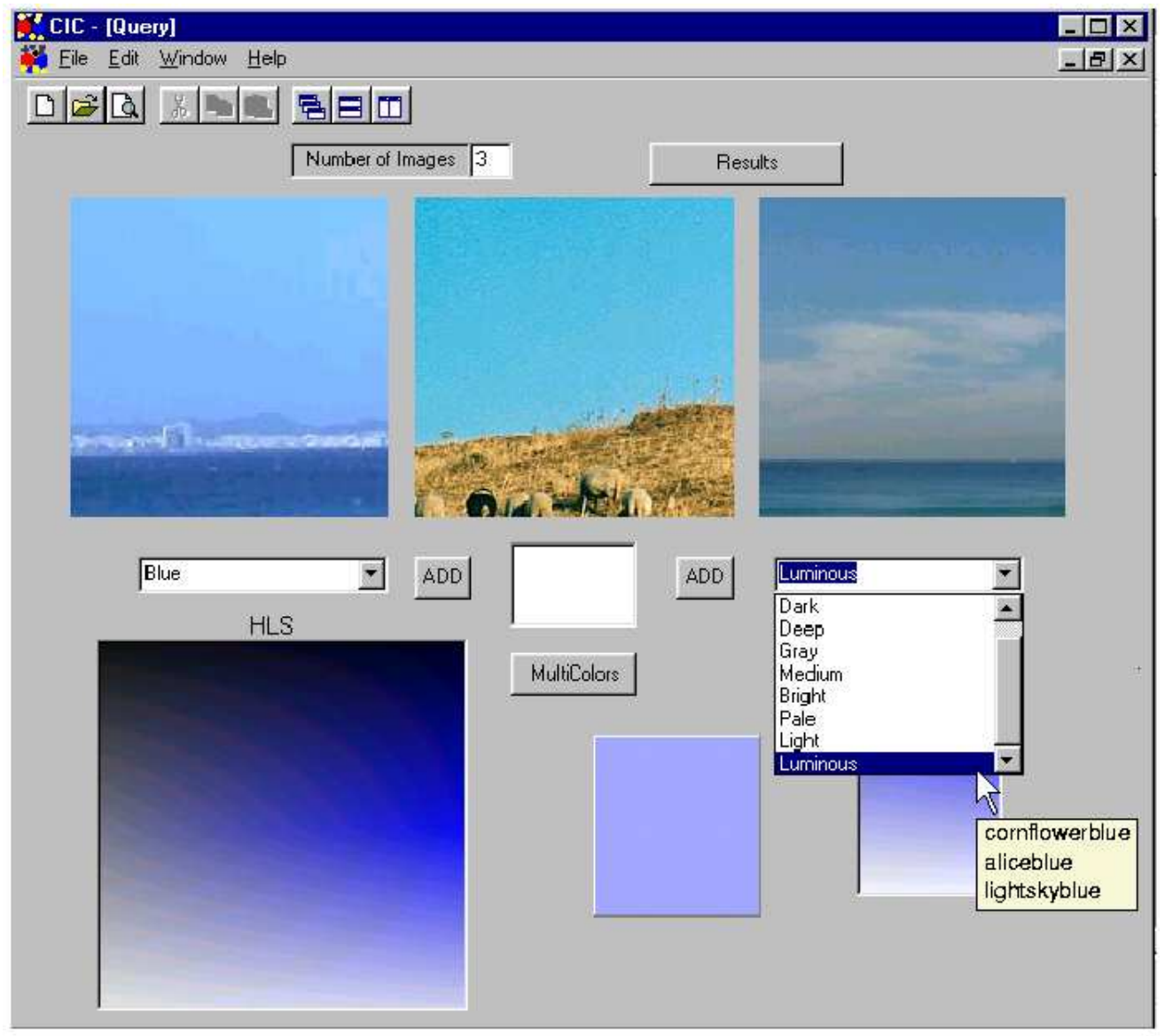

Fig. 12 Query with a color and a qualifier.

$$
\text { Precision }=\frac{\text { relevant retrieved }}{\text { all retrieved }}=\frac{\mathrm{RR}}{\mathrm{RR}+\mathrm{RNR}}
$$

Besides, the choice of image database is important to validate the software. We have used a colored image database containing a description of the images by keywords, like "the_color": the ImageBank (http://creative. gettyimages.com/imagebank/). The two rates presented were calculated (according to ImageBank).

On a total of 1000 images we have obtained the following results: $85 \%$ for the first rate (recall) and $89 \%$ for the second (precision) (cf. table 3).

\begin{tabular}{c|c|c} 
& Relevant & Not Relevant \\
\hline Retrieved & 897 & 103 \\
\hline Not retrieved & 164 & $/$ \\
\hline
\end{tabular}

Table 3 The validation results.

The reason why 103 images are not "well-classified" and 164 images are "not selected" is due to the subjectivity of the classification of ImageBank (IB) experts.
Our software permits to associate more dominant colors to an image than the IB does. Indeed, IB experts usually associate at most two color-keywords. Moreover, the naming of the colors is very subjective: for example, an expert may call a dark pink what another expert may call a magenta. This perception also depends a lot on the neighboring colors in the image: e.g. a yellow on a black background may not be perceived the same way than a yellow on a white background. This problem of perception is handled in section 4.4.

As an example, for green color all the relevant images according to ImageBank are presented in figure 14 . The figure 15 presents the green images selected by our software. The image (20) is not retrieved by our software (it is classified as a yellow image) and the images (21) and (22) are falsely retrieved (according to the IB classification). The images (21) and (22) are indexed by IB with only one color: respectively blue and pink. It should be noted that there is no order between images, i.e. in figure 15, we have kept the order the images appeared in the IB database. Indeed, IB indexing doesn't specify any order.

The classification obtained with this software is useful on, at least, two levels. On the first level, a classification by dominant color helps to find an image more 


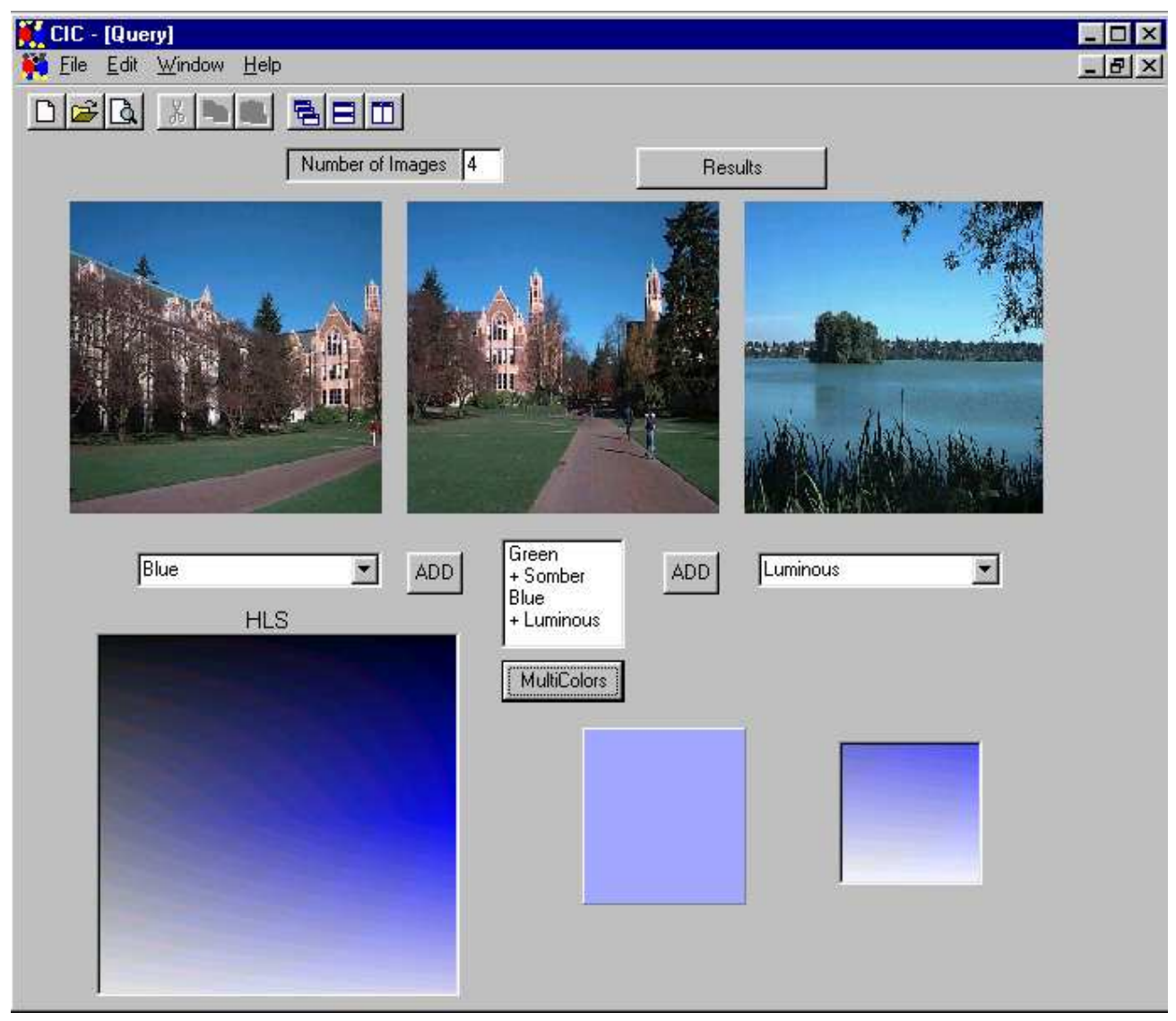

Fig. 13 Multi-color query.

easily, since color is one of the features that the human brain remembers the most [23]. On the second level, that should be called a "meta-level", this classification may become more meaningful since it allows a discrimination such as harmonious/non-harmonious images, indoor/outdoor images... i.e. a discrimination based on color exclusively. Defining a set of usual harmonies, such as 2-color, 3-color, mono-color... harmonies, it is easy to determine whether an harmony is more or less present in the image or not.

\subsection{Improving the processing}

In the presented processing, some problems due to the nature of the colored images are not taken into account. For example, some pixels can be considered as aberrant (in the HLS space). Indeed, we distinguish two kinds of problems:

1. Pixels around the edges are often aberrant, their colors are unexpected and they can be considered as noise (cf. figure 16) [4].

2. In uniform zones (in terms of perception of the color), one can have some aberrant pixels due to the digitization of the image, to the compression, etc. (cf. figure 17).
To solve these problems, we propose for the first one to use an edge detection method, and all the "edgepixels" will be eliminated before the processing.

Edges in images are areas with strong intensity contrasts - a jump in intensity from one pixel to the next. There are many ways to perform edge detection. However, the majority of different methods may be grouped into two categories, gradient and Laplacian. Gradient methods detect the edges by looking for the maximum and minimum in the first derivative of the image, while Laplacian methods search for zero crossings in the second derivative of the image. The chosen method is the "canny algorithm" [6] which uses the gradient method.

For the second problem, we have to determine all the uniform zones thanks to an edge detection, and then assign to each one a dominant color. For this purpose we have three possibilities:

- In each zone we consider a representative sub-zone like a center of gravity, the dominant color of the zone is then given by the average color of the pixels belonging to the sub-zone.

- In each zone we randomize two or three sub-zones. For each sub-zone we have an average color, the dominant color of the zone is given by the average of these colors. 
- In each zone we randomize a subset of pixels. This subset must contain $\alpha \%$ of the zone (for example, $\alpha=10$ ). The dominant color of the zone is given by the average color of the subset.

This work on zones is still in progress: we are testing different kinds of edge detectors and other methods for the representative color of a zone.

A last point to be studied is the computation of function $F_{t}(I)$ that is performed by an equi-weighted mean, i.e. each pixel has the same importance (cf. $\S 3$ ). To improve the classication, we shall assign different weights to the pixels. More precisely, at the beginning, every pixel gets the same weight and, depending on the pixels this weight may be decreased. For example isolated pixels or zones shall get a weaker weight and aberrant pixels (edge pixels) shall get a zero-weight.

\subsection{Towards a learning process}

Another improvement of the software is the handling of the perception. As color is a very subjective concept [5], we want to propose a tool for image retrieval that is perception-dependent. According to the user's eye sensibility, we suggest to modify the definition of the association color/hue (cf. figure 3), using a tool that learns the user's perception of colors, such as the one described in [28]. We propose a learning process for a colorimetric application. The method consists in memorizing the meaning of linguistic expressions associated to the colors, according to a learning done thanks to the user. To perform that, we use a graph and some linguistic modifiers (such as "much more", "a bit less", etc.) in order to store the acquired knowledge and its associated nuance [28]. For example, if the user wishes to learn "much more" as being "a bit more", then the process that underlies the linguistic expression "much more" is changed into the process underlying the expression "a bit more".

As we have seen in section 3, nine colors have been chosen and defined. But, this number can change according to the user's wishes. For example, he can add a new color mustard between green and yellow or he can delete a color: pink + magenta become pink.

In order to completely adapt the software, we shall also offer the user the possibility to change the definitions of the fundamental qualifiers (cf. figure 5).

For the dimension $\mathrm{H}$, the user will have the possibility to build his own membership functions $\left(f_{t} \forall t \in \mathcal{T}\right)$. Starting from the initial functions defined in section 3 , we will ask the user to modify the function parameters through linguistic modifiers used in [28], such as "green a little bit more bluish" instead of the initial green.

\section{Conclusion}

In this work we have developed an approach that permits to classify images according to their dominant color(s) and giving satisfactory results. We limit ourselves to fundamental colors and nine color qualifiers; those can be widened without modifying the approach, using a learning process according to the user's perception.

For the image extraction from the database, it is not necessary to browse all the 96 classes defined in section 4. We can make a first selection through the fundamental colors and then look at the corresponding son classes to refine the query.

In the database images are stored with their profiles, thus we extend this approach to look for images which don't have a given color or a set of colors.

To increase the accuracy of the image profile determination, we first detect homogeneous zones in the images and then we assign (a) dominant color(s) to each zone.

As a future work, more tests are required to improve the zone detection and the way to determine their dominant color(s). Moreover, our software will be proposed for medical applications: it will help medical forecasts and analysis, like tumor detection. It will retrieve images which contain zones with a strongly dominant color (for example red) which can correspond to cancerous cells. Our software will also be used to give a meaning to the classification, for example, indoor/outdoor images, or harmonious/disharmonious images...

\section{References}

1. A. Barla, F. Odone and A. Verri, Histogram intersection kernel for image classification, Proceedings of the International Conference on Image Processing (ICIP03), Barcelona, september 2003.

2. E. Binaghi, I. Gagliardi and R. Shettini, Image retrieval using fuzzy evaluation of color similarity, Pattern recognition and artificial intelligence, vol. 8, pp. 945-968, 1994.

3. B. Bouchon-Meunier, La Logique Floue et ses Applications, Addison-Wesley, 1995.

4. S. Bourghorbel, N. Boujemaa and C. Vertan, Histogrambased color signatures for image indexing, Proceedings of Information processing and management of uncertainty in knowledge-based systems, IPMU, 2002.

5. C. Boust, H. Chahine, F. Viénot, H. Brettel, M. Ben Chouikha and G. Alquié, Color Correction Judgements of Digital Images by Experts and Naive Observers, Proceedings of PICS 2003, pp. 4-9, Rochester, NY, USA, 2003.

6. J. Canny, A Computational Approach to Edge Detection, IEEE Transactions on Pattern Analysis and Machine Intelligence, vol. 8, no. 6, Nov 1986.

7. Y. Chen and J. Z. Wang, A Region-Based Fuzzy Feature Matching Approach to Content-Based Image Retrieval, IEEE Transactions on Pattern Analysis and Machine Intelligence, vol. 24, no. 9, pp. 1252-1267, 2002.

8. E. Claridge, S. Cotton, P. Hall and M. Moncrieff, From colour to tissue histology: Physics-based interpretation of images of pigmented skin lesions, Medical Image Analysis, vol. 7, no. 4, pp. 489-502, 2003.

9. J.P. Couwenbergh, Guide complet et pratique de la couleur, Paris, Eyrolles, 2003. 
10. D.C. Durairaj, M.C. Krishna and R. Murugesan, Integration of color and boundary information for improved region of interest identification in electron magnetic resonance images, Computerized Medical Imaging and Graphics, vol. 28, Issue 8, pp. 445-452, December 2004.

11. L. Foulloy, Du contrôle symbolique des processus : démarche, outils, exemples, Ph.D. Thesis, Université Paris XI, September 1990.

12. H. Frigui, Interactive image retrieval using fuzzy sets, Pattern Recognition Letters, Vol. 22, pp: 1021-1031, 2001.

13. M. Hammami, L. Chen, D. Zighed and Q. Song, Définition d'un modèle de peau et son utilisation pour la classification des images, Proceedings of MediaNet'2002, pp. 187-198, Sousse, Tunisia, June 2002.

14. J. Han and K.K. Ma, Fuzzy color histogram and its use in color image retrieval, IEEE Transactions on Image Processing, Vol. 11 (8), pp. 944-952, 2002.

15. F. Herrera and L. Martínez, A model based on lingustic two-tuples for dealing with multigranularity hierarchical linguistic contexts in multiexpert decision-making, IEEE Transactions on Systems, Man and Cybernetics, Part B, vol. 31, no. 2, pp. 227-234, 2001.

16. L. Hildebrand and B. Reusch, Fuzzy Color Processing, in: Book Kerre, E.; Nachtegael, M.; (Editors) Fuzzy Techniques in Image Processing, Studies in Fuziness and Soft Computing, Vol. 52, Physica-Verlag, Heidelberg, 2000.

17. P. Hong, T. Qi and T.S. Huang, Incorporate support vector machines to content-based image retrieval with relevance feedback, Proceedings of IEEE International Conference on Image Processing, Vancouver, Canada, 2000.

18. B. Le Saux, Classification non exclusive et personnalisation par apprentissage : Application à la navigation dans les bases d'images, Ph.D. Thesis, INRIA, France, July 2003.

19. J.-F. Omhover, M. Detyniecki and B. Bouchon-Meunier, A Region-Similarity-Based Image Retrieval System, Proceedings of IPMU'04, pp. 1461-1468, Perugia, Italy, July 2004.

20. E. Petrakis and S. Orphanoudakis, Methodology for the representation, indexing and retrieval of images by content, Image and Vision Computing, vol. 11, no. 8, pp. 504-521, 1993.

21. J. Roire, Les noms des couleurs, Pour la science, Hors série, no. 27, 2000.

22. G. Salton and M.J. McGill, Introduction to modern Information Retrieval, McGraw-Hill, 1983.

23. S. Shoemaker, Colors, Subjective Relations and Qualia, Philosophical Issues, no. 7, pp. 55-66, 1996.

24. N. Sugano, Color-naming system using fuzzy set theoretical approach, The 10th IEEE International Conference on Fuzzy Systems, Vol. 1, pp: 81-84, 2001.

25. M.J. Swain and D.H. Ballard, Color indexing, IJCV, vol. 7, no. 1, pp. 11-32, 1999.

26. I. Truck, H. Akdag and A. Borgi, A Symbolic Approach for Colorimetric Alterations, Proceedings of EUSFLAT 2001, 105-108, Leicester, England, September 2001.

27. I. Truck, Approches symbolique et floue des modificateurs linguistiques et leur lien avec l'agrégation, Ph.D. Thesis, Université de Reims Champagne-Ardenne, France, December 2002

28. I. Truck and H. Akdag, Supervised Learning using Modifiers: Application in Colorimetrics, Proceedings of the ACS/IEEE International Conference on Computer Systems and Applications (AICCSA'03), pp. 116 (7 pages), Tunisia, July 2003.

29. V. Vapnik, Statistical learning theory, John Wiley and Sons, New York, 1998.

30. C. Vertan and N. Boujemaa, Embedding Fuzzy Logic in Content Based Image Retrieval, Proceedings of the 19th International Meeting of the North American Fuzzy Information Processing Society NAFIPS 2000 Atlanta, July 2000.

31. C. Vertan and N. Boujemaa, Using fuzzy histograms and distances for color image retrieval, Challenges of Image Retrieval, Brighton CIR'2000, pp. 1-6, 2000.

32. J.Z. Wang and Y. Du, Scalable Integrated Region-Based Image Retrieval Using IRM and Statistical Clustering, Proceedings of the ACM and IEEE Joint Conference on Digital Libraries, pp. 268-277, Roanoke, VA, June 2001. 


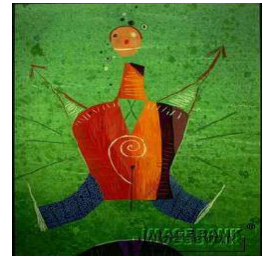

(1)

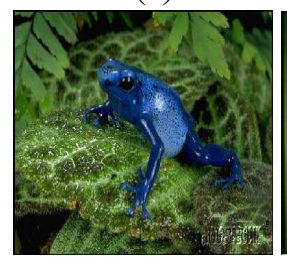

(6)

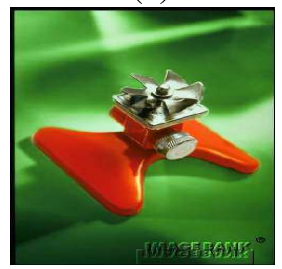

(11)

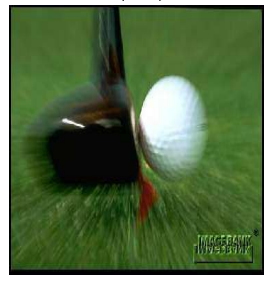

(16)

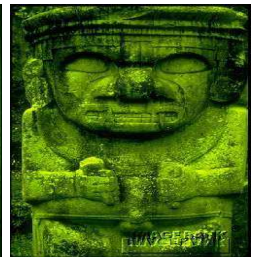

(2)

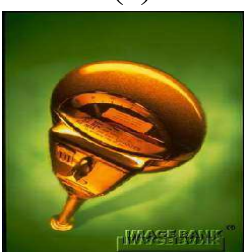

(7)

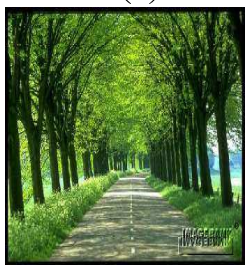

(12)

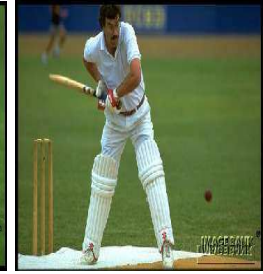

(17)

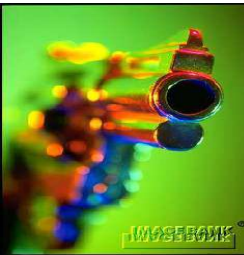

(3)

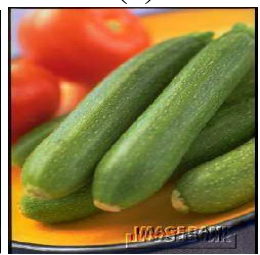

(8)

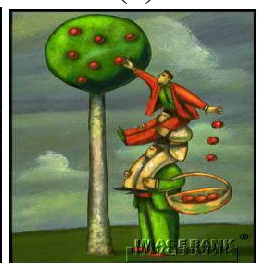

(13)

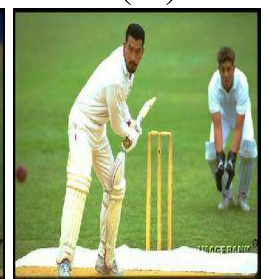

(18)

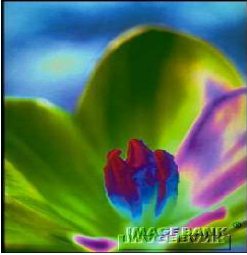

(4)

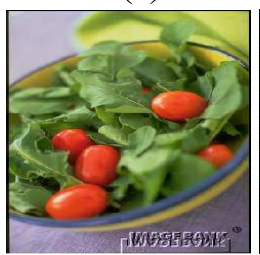

(9)

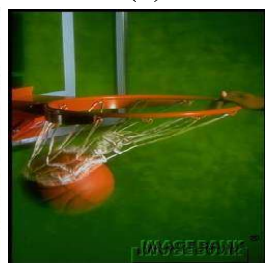

(14)

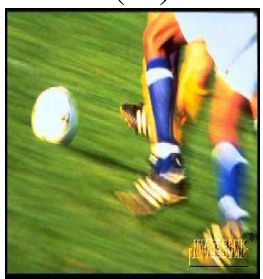

(19)

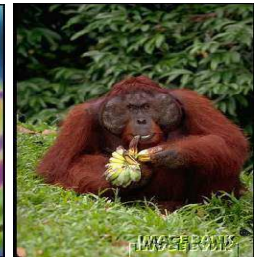

(5)

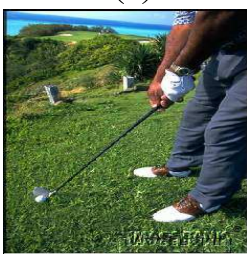

(10)

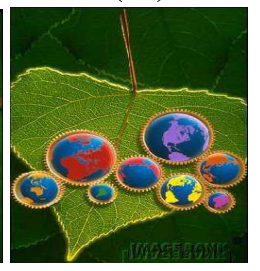

(15)

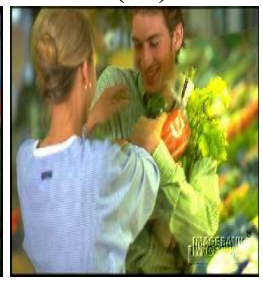

(20)

Fig. 14 Green images according to ImageBank. 


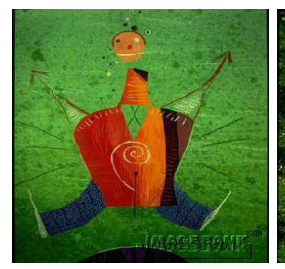

(1)

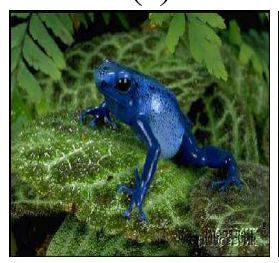

(6)

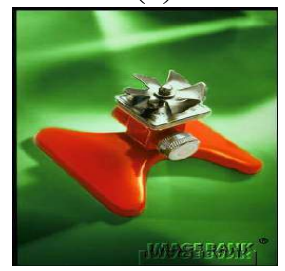

(11)

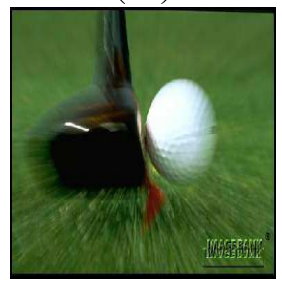

(16)

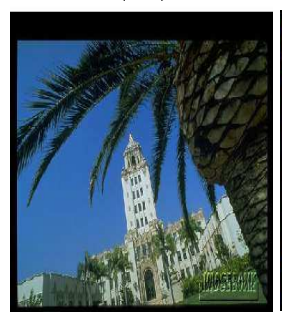

(21)

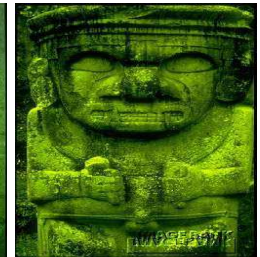

(2)

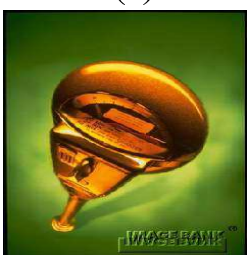

(7)

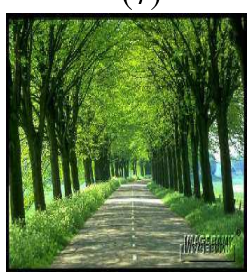

(12)

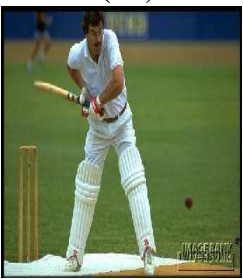

(17)

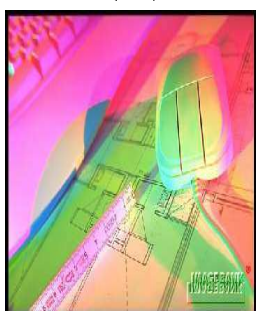

(22)

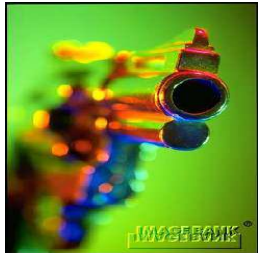

(3)

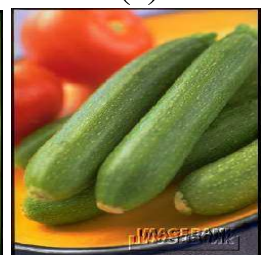

(8)

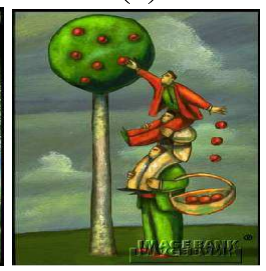

(13)

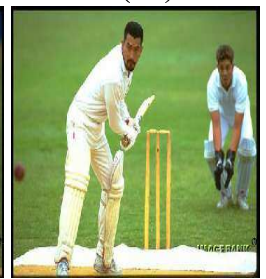

(18)

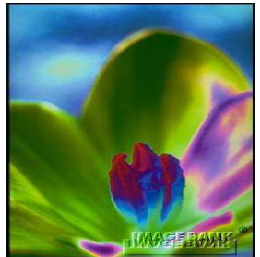

(4)

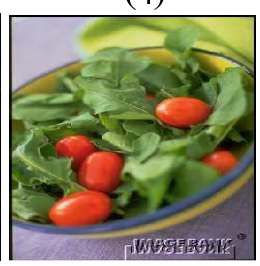

(9)

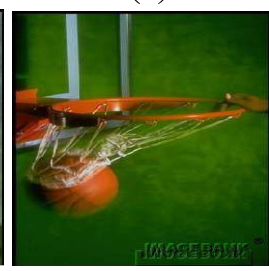

(14)

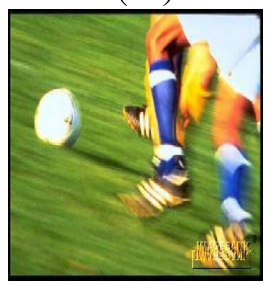

(19)

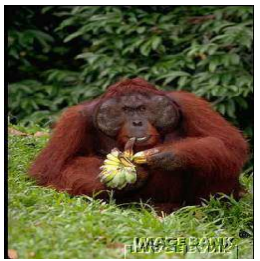

(5)

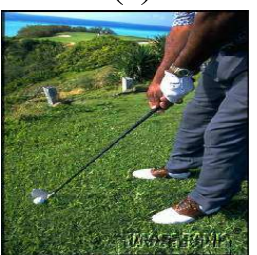

(10)

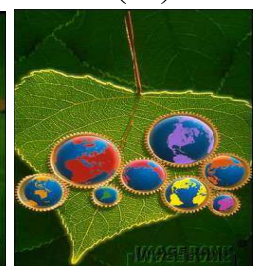

(15)

Fig. 15 Green images retrieved by our software.
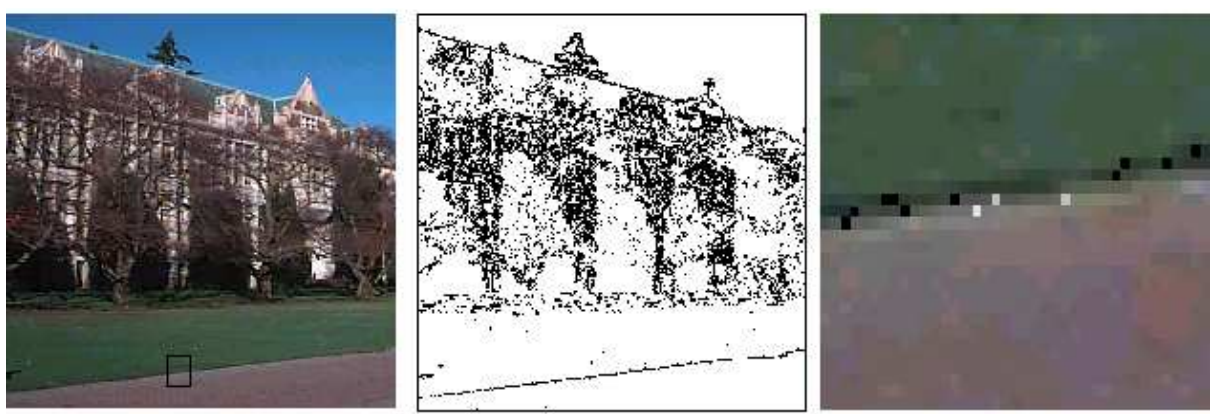

Fig. 16 Edge detection and "noise pixels" around an edge. 

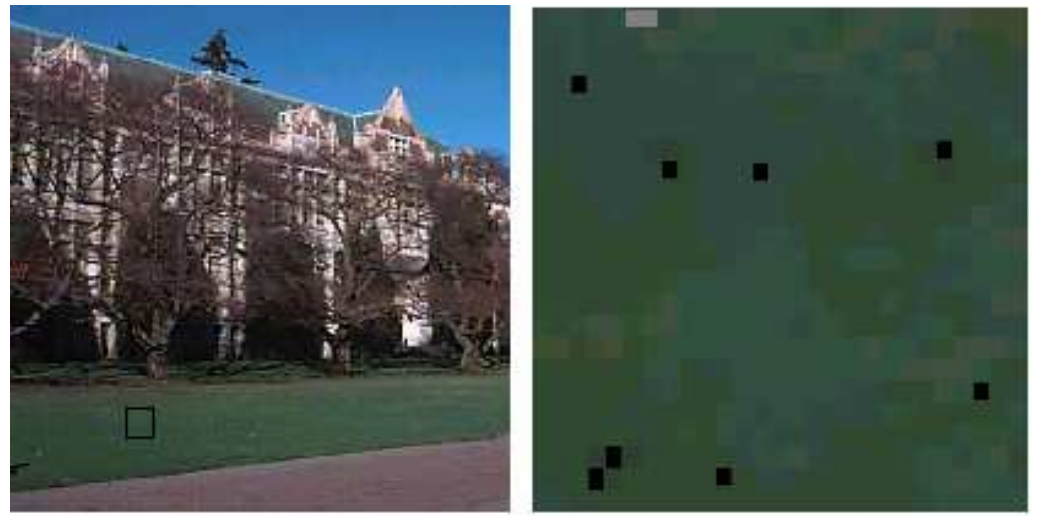

Fig. 17 Uniform zone 